\title{
Alterations of protein glycosylation in embryonic stem cells during adipogenesis
}

\author{
WENGUANG LIU*, XINGRONG YAN*, WEI LIU, YANGYANG WANG, YANG RAO, HANJIE YU, \\ JIHONG CUI, XIN XIE, MEI SUN, LU YIN, HONGMIN LI and FULIN CHEN
}

Faculty of Life Sciences, Northwest University, Xi'an, Shaanxi 710069, P.R. China

Received May 19, 2016; Accepted October 25, 2017

DOI: $10.3892 /$ ijmm.2017.3240

\begin{abstract}
The understanding of adipose tissue development is crucial for the treatment of obesity-related diseases. Adipogenesis has been extensively investigated at the gene and protein levels in recent years. However, the alterations in protein glycosylation during this process remains unknown, particularly that of parthenogenetic embryonic stem cells (pESCs), a type of ESCs with low immunogenicity and no ethical concerns regarding their use. Protein glycosylation markedly affects cell growth and development, cell-to-cell communication, tumour growth and metastasis. In the present study, the adipogenic potentials of J1 ESCs and pESCs were first compared and the results demonstrated that pESCs had lower adipogenic potential compared with J1 ESCs. Lectin microarray was then used to screen the alteration of protein glycosylation during adipogenesis. The results revealed that protein modification of GlcNAc and $\alpha-1-2$-fucosylation increased, whereas $\alpha-1-6$-fucosylation, $\alpha-2-6$-sialylation and $\alpha$-1-6-mannosylation decreased in J1 ESCs and pESCs during this process. In addition, $\alpha-1-3$-mannosylation decreased only in pESCs. Lectin histochemistry and quantitative polymerase chain reaction of glycosyltransferase confirmed the results obtained by lectin microarray. Therefore, protein glycosylation of ESCs was significantly altered during adipogenesis, indi-
\end{abstract}

Correspondence to: Dr Jihong Cui, Faculty of Life Sciences, Northwest University, 229 Taibai North Road, Xi'an, Shaanxi 710069, P.R. China

E-mail: cjh@nwu.edu.cn

*Contributed equally

Abbreviations: pESCs, parthenogenetic embryonic stem cells; ESCs, embryonic stem cells; WGA, wheat germ agglutinin; ConA, concanavalin A; NPA, Narcissus pseudonarcissus; HHL, hippeastrum hybrid lectin; GNA, Galanthus nivalis; MSCs, pluripotent mesenchymal stem cells; RA, retinoic acid; c/ebp, CCATT/enhancer-binding protein; ppar $\gamma$, peroxisome proliferator-activated receptor $\gamma$; SSEA, stage-specific embryonic antigen

Key words: adipogenesis, embryonic stem cells, glycosylation, lectin microarray, parthenogenetic embryonic stem cells cating that protein glycosylation analysis is not only helpful for studying the mechanism of adipogenesis, but may also be used as a marker to monitor adipogenic development.

\section{Introduction}

The increase of the adipogenic cell population and accumulation of lipid may lead to obesity. Obesity and related diseases, including type 2 diabetes, stroke, hypertension and cardiovascular disease, have become major medical problems in modern society $(1,2)$. To control obesity and related diseases, it is crucial to understand and monitor cellular adipogenic lineage differentiation and development.

Embryonic stem cells (ESCs) (3), mesenchymal stem cells (MSCs) (4) and preadipocytes (5) have been used to study cellular adipogenic differentiation in vitro. Key factors regulating adipogenesis have been extensively investigated at the gene and protein levels in recent years. Adipogenesis may be divided into two stages, namely commitment and terminal differentiation. Commitment has been defined as the process leading from multipotent stem cells to preadipocytes (4). Certain factors, including bone morphogenetic proteins 2, 4, Wnt and retinoic acid (RA), have been identified as activators of the commitment pathway $(4,6,7)$, whereas sonic hedgehog inhibits this process $(8,9)$. RA was found to play an important role in the early differentiation of ESCs, and RA-pretreated embryoid bodies (EBs) displayed a high level of adipogenesis (3). Terminal differentiation has been defined as cell differentiation from preadipocytes to adipocytes. In vitro, terminal differentiation may be induced by a cocktail containing insulin-like growth factor-1 (IGF-1) (10), glucocorticoid and cyclic AMP (cAMP) (11). During the process of terminal differentiation, the expression of several key transcription factor families, such as CCATT/enhancer-binding protein (c/ebp) family (12) and the peroxisome proliferator-activated receptor (ppar) family, was found to be upregulated. The coordination of these transcription factors produces the adipocyte phenotype and maintains the expression of adipocyte genes (13).

Protein glycosylation is an enzymatic process that produces glycosidic linkages of glycans to proteins, and it is a ubiquitous post-translational modification. Approximately $70 \%$ of human proteins contain $\geq 1$ glycan chains (14) and $1 \%$ of the human genome is involved in glycan production and modification (15). Protein glycosylation plays a crucial role in cell growth and 
development $(15,16)$, cell-to-cell communication $(17,18)$, tumour growth and metastasis $(19,20)$. Indeed, several stemness markers, such as stage-specific embryonic antigen (SSEA)-1 and -3 , are glycoproteins (21). Although adipogenesis has been studied at the gene and protein level, it remains unknown how protein glycosylation is altered during this process, particularly in parthenogenetic ESCs (pESCs), which are obtained from parthenogenetic blastocysts, exhibit low immunogenicity and are assosiated with no ethical issues regarding their use.

Lectin microarray is a technology for screening the structure of the glycome, where a large number of lectins with various glycan-binding specificities are printed on a microarray. This technology enables rapid and high-throughput profiling of protein glycosylation without liberation of glycans from proteins. In addition, altered glycan patterns may be visualised and located in tissues and cells through lectin histochemistry (22). Lectin mircoarray has been applied mainly to detect glycans as disease-related biomarkers $(23,24)$. Tateno et al compared protein glycosylation conditions of human ESCs and somatic cells, and found that rBC2LCN [binding for Fuca12Galß1-3GlcNAc (GalNAc)-containing glycans] signaling only existed in undifferentiated ESCs, but not in differentiated somatic cells. This result indicated that $\mathrm{rBC} 2 \mathrm{LCN}$ was the optimal lectin probe for evaluating pluripotency, and that the presence of Fuc $\alpha 1-2 \mathrm{Gal} / 1-3 \mathrm{GlcNAc}$ (GalNAc)-containing glycans was a biomarker of pluripotency.

In the present study, the alterations of J1 ESCs and pESCs during adipogenesis were analysed using a lectin microarray. The basic properties and differentiation potential of the two types of ESCs were first compared, and it was observed that J1 ESCs and pESCs have different adipogenic potentials. The alterations of protein glycosylation in J1 ESCs and pESCs during this process were then compared. This experiment is not only helpful for the study of the mechanism of adipogenesis, but may also indicate new markers for monitoring adipogenic development.

\section{Materials and methods}

Cell cultivation and characterisation. J1 ESCs and pESCs (from C57BL/6J mice, a kind gift from Professor Jinlian Hua, the Northwest A\&F University, Shaanxi, China) were cultured on a feeder layer of mitomycin C (Sigma-Aldrich, Merck KGaA, St. Louis, MO, USA)-treated mouse embryonic fibroblasts with ESGRO Complete PLUS Clonal Grade Medium (Millipore, Billerica, MA, USA) (25) in a $37^{\circ} \mathrm{C} / 5 \% \mathrm{CO}_{2}$ incubator. The cells were passaged every 4-5 days using Accutase (Millipore) at a ratio of 1:3-1:6 and observed under a phase contrast microscope (Nikon, Tokyo, Japan).

To assess cellular pluripotency in vitro, J1 ESCs and pESCs grown on glass coverslips were fixed with $4 \%$ cold paraformaldehyde (Sigma-Aldrich, Merck KGaA) in phosphate-buffered saline (PBS) for $30 \mathrm{~min}$, and then permeabilized with $0.1 \%$ Triton X-100 (Sigma-Aldrich, Merck $\mathrm{KGaA}$ ) in PBS for 8 min. Fixed cells were blocked with $5 \%$ bovine serum albumin (BSA; Sigma-Aldrich, Merck KGaA) in PBS for $1 \mathrm{~h}$ and incubated overnight at $4^{\circ} \mathrm{C}$ with primary antibodies including rabbit anti-Nanog (M-149, sc-33760), rabbit anti-Oct4 (H-134, sc-9081) and mouse anti-SSEA-1 (480, sc-21702) (dilution 1:200; all from Santa Cruz Biotechnology,
Inc., Santa Cruz, CA, USA). Secondary antibodies (A-11034 and A-21044, dilution 1:300; Invitrogen Life Technologies, Thermo Fisher Scientific, Carlsbad, CA, USA) were added and allowed to incubate for $1 \mathrm{~h}$ at $37^{\circ} \mathrm{C}$. The nuclei were counterstained with 4',6-diamidino-2-phenylindole (DAPI; Sigma-Aldrich, Merck KGaA). The cells were washed thoroughly with PBS after each incubation step. The images were captured using a FV1000 confocal microscope (Olympus, Tokyo, Japan).

Animals. Five-week-old nude mice were obtained from the Experimental Animal Center of the Fourth Military Medical University. All the animal studies and protocols were processed according to the guidelines of the Animal Holding Unit of Northwest University. To test pluripotency in vivo, J1 ESCs and pESCs were dispersed using Accutase. Cells $\left(1 \times 10^{6}\right)$ in $50 \mu 1$ Dulbecco's modified Eagle's medium (DMEM; Hyclone, Logan, UT, USA) were subcutaneously injected into nude mice. The animals were sacrificed and the formed teratomas were harvested 4 weeks after the injections. The specimens were fixed with $4 \%$ paraformaldehyde, embedded in paraffin, sectioned at $4 \mu \mathrm{m}$, and stained with hematoxylin and eosin.

J1 ESC and pESC proliferation was analyzed using the cell proliferation reagent 4-[3-(4-iodophenyl)-2(4-nitrophenyl)-2H-5-tetrazolio]-1,3-benzene disulfonate (WST-1) assay (Roche Diagnostics, Mannheim, Germany). For this procedure, 2,000 cells in $100 \mu \mathrm{l}$ medium were seeded into 96-well plates in triplicate. WST-1 (10 $\mu \mathrm{l})$ was added to each well and incubated for up to 3 days. The absorbance was measured every $24 \mathrm{~h}$ with a microplate reader (Synergy HT; BioTek Instruments, Inc., Winooski, VT, USA) at $450 \mathrm{~nm}$, according to the manufacturer's instructions.

Mesoderm differentiation of J1 ESCs and pESCs. EBs were generated using the hanging drop method (3). Cells were dissociated with Accutase to obtain single-cell suspensions. Hanging drops containing $1 \times 10^{3}$ cells in $20 \mu$ medium [DMEM supplemented with $50 \mathrm{mg} / \mathrm{ml} \mathrm{L-proline} \mathrm{(Sigma-Aldrich,}$ Merck KGaA), $1 \%$ non-essential amino acids (Millipore), $1 \%$ 2-mercaptoethanol (Sigma-Aldrich, Merck KGaA) and $20 \%$ fetal bovine serum (FBS; Gibco Life Technologies, Thermo Fisher Scientific, Grand Island, NY, USA)] were maintained for 2 days on the lids of non-adherent dishes filled with PBS. The formed EBs were then transferred into non-adherent plates in medium with or without $10^{-5} \mathrm{mM}$ RA (Sigma-Aldrich, Merck KGaA) for a further 3 days.

For osteogenic and chondrogenic differentiation, non-RA-pretreated EBs were trypsinized and plated on cell culture dishes coated with $0.1 \%$ gelatin (Sigma-Aldrich, Merck KGaA). The cells were cultured in osteogenic induction medium [DMEM supplemented with $50 \mathrm{nM}$ dexamethasone, $10 \mathrm{mM} \beta$-glycerophosphate and $50 \mu \mathrm{M}$ ascorbate-2-phosphate (all from Sigma-Aldrich, Merck KGaA) and 10\% FBS] and in chondrogenic induction medium [DMEM supplemented with $50 \mu \mathrm{M}$ ascorbic acid 2-phosphate, $10 \mathrm{ng} / \mathrm{ml}$ transforming growth factor- $\beta 1$ and $500 \mathrm{ng} / \mathrm{ml}$ IGF (R\&D Systems, Inc., Minneapolis, MN, USA) and 10\% FBS] for 20 days. The medium was changed every 3 days and the specimens were stained with Alizarin Red and Safranin O, respectively. To detect osteogenic and chondrogenic differentiation-related gene expression, total RNA was extracted from cells after 
induction with RNA Isolation reagent (Takara Bio, Inc., Tokyo, Japan) according to the manufacturer's instructions. The extracted RNA was quantified using a GeneQuant pro (GE Healthcare Life Sciences, Logan, UT, USA). The RevertAid $^{\mathrm{TM}}$ First Strand cDNA Synthesis kit (Thermo Fisher Scientific, Inc., Waltham, MA, USA) was used to convert the RNA template into cDNA. The reaction was conducted in an Eppendorf Mastercycler (Eppendorf, Hamburg, Germany) as follows: $65^{\circ} \mathrm{C}$ for $5 \mathrm{~min}, 25^{\circ} \mathrm{C}$ for $5 \mathrm{~min}, 50^{\circ} \mathrm{C}$ for $50 \mathrm{~min}$ and $70^{\circ} \mathrm{C}$ for $15 \mathrm{~min}$. The amplified products were separated with agarose gels and visualized with the assistance of ethidium bromide and ultraviolet light. The polymerase chain reaction (PCR) primers are listed in Table I.

For adipogenic differentiation, RA-pretreated EBs were trypsinized and plated onto gelatin-coated plates and cultured in adipogenic induction medium [DMEM supplemented with $1 \mu \mathrm{M}$ dexamethasone, $200 \mu \mathrm{M}$ indomethacin and $10 \mu \mathrm{g} / \mathrm{ml}$ insulin (both from Sigma-Aldrich, Merck KGaA), $0.5 \mathrm{mM}$ methylisobutylxanthine (MP Biomedicals LLC, Santa Ana, CA, USA) and 10\% FBS] for 20 days. The medium was changed every 3 days. At 5, 10, 15 and 20 days after induction, the specimens were processed for quantitative PCR (qPCR) to evaluate the expression of adipogenic differentiation-related genes, followed by immunofluorescence staining of c/ebp $\alpha$ and fabp4 (dilution 1:200; both from Santa Cruz Biotechnology, Inc.), as described above. The specimens were further stained with Oil red $\mathrm{O}$. The proportion of cells undergoing adipogenic differentiation was calculated by counting Oil red O-positive and -negative cells using a microscope. Five fields of view were counted for each specimen, as previously described (26). The qPCR primers are listed in Table I.

Lectin microarrays and lectin histochemistry. A lectin microarray of 37 lectins (Vector Laboratories, Burlingame, CA, USA; Sigma-Aldrich, Merck KGaA and Calbiochem-Merck Co., Darmstadt, Germany) was produced as previously described (22). The protein was extracted from specimens 20 days after adipogenic induction with T-PER reagent (Pierce Biotechnology Inc., Rockford, IL, USA), according to the manufacturer's instructions. The extracted protein was labelled by a $\mathrm{Cy} 3$ fluorescent dye and purified by Sephadex G-25 columns (both from GE Healthcare Life Sciences). Subsequently, $4 \mu \mathrm{g}$ of Cy3-labeled protein diluted with $0.5 \mathrm{ml}$ of hybridization buffer (2\% BSA, $500 \mathrm{Mm}$ glycine and $0.1 \%$ Tween-20 in PBS) was applied to the blocked lectin microarrays, and the array was then incubated at $37^{\circ} \mathrm{C}$ at $2.6 \times 10^{-3} \times \mathrm{g}$ for $3 \mathrm{~h}$. The slides were washed three times with PBST (0.2\% Tween-20 in PBS), then washed with PBS, and dried by centrifugation at $60 \mathrm{x} \mathrm{g}$ for $5 \mathrm{~min}$. The microarrays were then scanned with a Genepix 4000B confocal scanner (Axon Instruments, Union City, CA, USA) using a 70\% photomultiplier tube and $100 \%$ laser power. The images were analysed at $532 \mathrm{~nm}$ for $\mathrm{Cy} 3$ detection using the GenePix 3.0 software (Axon Instruments). The mean background was subtracted, and the values lower than the mean background \pm 2 standard deviations (SD) were removed from each data point. For each lectin, the median of the effective data points was globally normalized to the sum of the medians for all the effective data points in one block. Each sample was consistently observed in three repeated slides. The normalized medians and the
Table I. List of primers used for RT-PCR/qPCR

\begin{tabular}{|c|c|c|}
\hline Genes & $\begin{array}{l}\text { Primer sequences, } \\
\text { sense/antisense }\left(5^{\prime}-3^{\prime}\right)\end{array}$ & $\begin{array}{l}\text { Product } \\
\text { size (bp) }\end{array}$ \\
\hline fabp4 & $\begin{array}{l}\text { ATGGCCAAGCCCAACATGAT } \\
\text { CTTCCTGTCGTCTGCGGTGAT }\end{array}$ & 135 \\
\hline $\operatorname{ppar} \gamma$ & $\begin{array}{l}\text { AAGGGTGCCAGTTTCGATCCGTAG } \\
\text { ATCAGGGAGGCCAGCATCGTGTAG }\end{array}$ & 154 \\
\hline alp & $\begin{array}{l}\text { ACAACCTGACTGACCCTTCG } \\
\text { AАTCCTGCСТCСТTCCACC }\end{array}$ & 104 \\
\hline ocn & $\begin{array}{l}\text { TGGCCATCACCCTGTCTCCT } \\
\text { GAGACCACTCCAGCACAACTCCT }\end{array}$ & 229 \\
\hline aggrecan & $\begin{array}{l}\text { TGTAAGGACTGTCTATCTACACGCC } \\
\text { TGGATGGTGATGTCGTCTTCAC }\end{array}$ & 147 \\
\hline $\begin{array}{l}\text { Type II } \\
\text { collagen }\end{array}$ & $\begin{array}{l}\text { AAGACGGTGAGACGGGAGCC } \\
\text { ACCATCAGTACCAGGAGTGCCAG }\end{array}$ & 287 \\
\hline oct 4 & $\begin{array}{l}\text { GATCACTCACATCGCCAATCA } \\
\text { CTGTAGCCTCATACTCTTCTCGTT }\end{array}$ & 128 \\
\hline c/ebp $\alpha$ & $\begin{array}{l}\text { AAGAGGACGAGGCGAAGCAGC } \\
\text { GCGCGATCTGGAACTGCAAGT }\end{array}$ & 132 \\
\hline Alg3 & $\begin{array}{l}\text { TACCTATCCCGCTCCTTTGAC } \\
\text { CCCTGTTCTGTGCCACCTAC }\end{array}$ & 171 \\
\hline Alg12 & $\begin{array}{l}\text { GGAGTCCTTGGGCTTGGTGTG } \\
\text { CAGGCTGTGAGGGCTGGTAAGAC }\end{array}$ & 191 \\
\hline$\beta$-actin & $\begin{array}{l}\text { CAGCCTTCCTTCTTGGGTAT } \\
\text { TGGCATAGAGGTCTTTACGG }\end{array}$ & 100 \\
\hline
\end{tabular}

RT-PCR, reverse transcription-polymerase chain reaction; qPCR, quantitative PCR; fabp4, fatty acid-binding protein 4 ; ppar $\gamma$, peroxisome proliferator-activated receptor $\gamma$; alp, alkaline phosphatase; ocn, osteocalcin; c/ebp $\alpha$, CCATT/enhancer-binding protein $\alpha$.

SD of each lectin from 9 repeated blocks were averaged. The normalized data were then compared to determine any relative changes in the protein glycosylation levels. The original data were further analysed using Expander 6.0 software (http:// acgt.cs.tau.ac.il/expander/).

Cy3-labeled lectins were also applied to detect the specific glycan structure present in the cells following the protocol previously described (27). Briefly, cells grown on glass coverslips were fixed with $4 \%$ paraformaldehyde in PBS for $30 \mathrm{~min}$, permeabilized with $0.1 \%$ Triton X-100 in PBS for $30 \mathrm{~min}$, and blocked with 5\% BSA in PBS for $1 \mathrm{~h}$. The cells were incubated with a solution containing a final concentration of $100 \mu \mathrm{g} / \mathrm{ml} \mathrm{Cy3}$ fluorescein-labeled lectins and 5\% (w/v) BSA for $3 \mathrm{~h}$ at room temperature in the dark. Subsequently, they were counterstained with $1 \mu \mathrm{g} / \mathrm{ml}$ DAPI and washed with PBS. Imaging was performed using a FV1000 confocal microscope (Olympus).

$q P C R$. The qPCR primers are listed in Table I. qPCR was performed in a MyiQ single-color detection system (Bio-Rad Laboratories, Inc., Richmond, CA, USA). The qPCR protocol was as follows: Hot start for $30 \mathrm{sec}$ at $95^{\circ} \mathrm{C}$, denaturation for $10 \mathrm{sec}$ at $95^{\circ} \mathrm{C}$, annealing for $15 \mathrm{sec}$ at $55^{\circ} \mathrm{C}$, and extension for $20 \mathrm{sec}$ at $72^{\circ} \mathrm{C}$ for $30-35$ cycles. Melt curve analyses were 
A

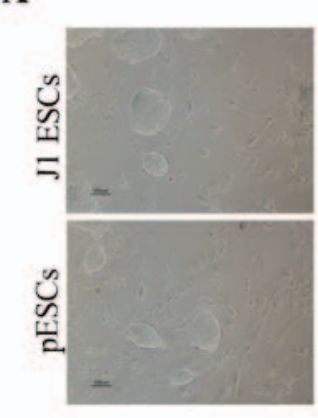

C

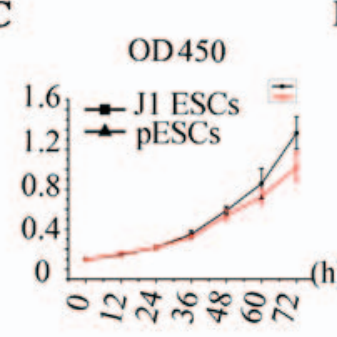

B

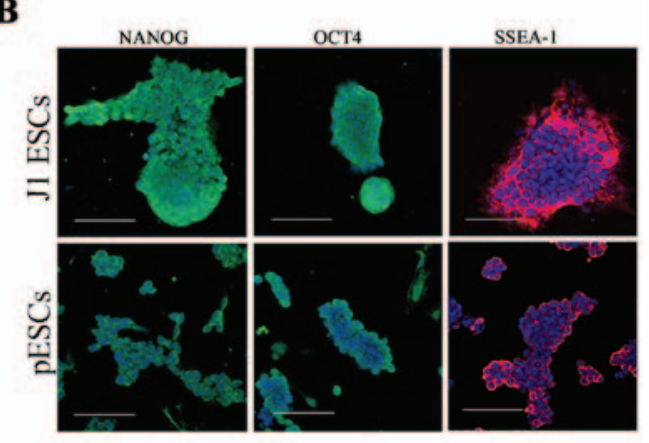

D

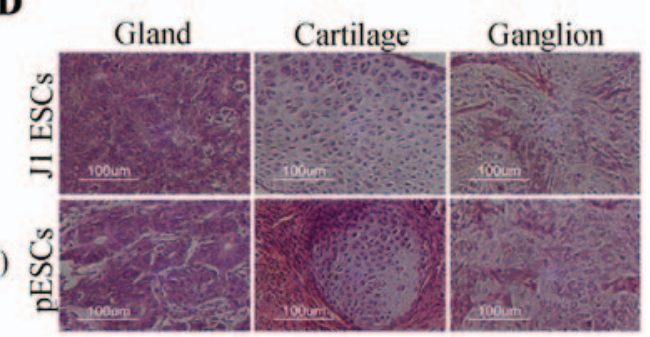

Figure 1. Basic characterization of J1 embryonic stem cells (ESCs) and parthenogenetic ESCs (pESCs) in vitro and in vivo. (A) Undifferentiated J1 ESCs and pESCs cultured on mouse embryonic fibroblasts formed colonies. (B) Immunofluorescence labeling of NANOG, octamer-binding transcription factor 4 (OCT4) and stage-specific embryonic antigen (SSEA)-1 in undifferentiated J1 ESC and pESC colonies. (C) Growth kinetics of J1 ESCs and pESCs (3 repeat/timepoint; data represent means \pm standard deviation). (D) Histological analysis differentiated elements of teratomas from J1 ESCs and pESCs 4 weeks after implantation in nude mice. Hematoxylin and eosin staining revealed that J1 ESCs as well as pESCs were able to form teratomas, containing ganglion (ectodermal), gland (endodermal) and cartilage (mesodermal) tissues. Scale bar, $100 \mu \mathrm{m}$. OD, optical density.

performed for each experiment to verify the formation of a single, desired PCR product. GAPDH was used as an internal control to normalize the amount of RNA. Absolute values obtained for each sample were normalized to the GAPDH signal. Single-peak melting profiles were obtained for the amplifications, and the size of the PCR product was confirmed by agarose gel electrophoresis. Each experiment was repeated three times. The $\Delta \Delta \mathrm{Ct}$ method was used to calculate relative amounts of transcripts.

Statistical analysis. Data are presented as mean \pm SD and compared using unpaired Student's t-test using SPSS statistics, version 18 (SPSS, Inc., Chicago, IL, USA). The statistical significance level was defined as $\mathrm{P}<0.05$.

\section{Results}

Basic properties of J1 ESCs and pESCs. Both J1 ESCs and pESCs had a high nucleus:cytoplasm ratio and exhibited compact colony structure during in vitro culture and expansion (Fig. 1A). Oct4 and Nanog are transcription factors located in the cell nucleus. Ssea-1 is a type of glycoprotein located on the cell membrane. According to the results presented in Fig. 1B, J1 ESCs and pESCs were strongly positive for immunofluorescence staining of these stemness factors. Both cell types proliferated rapidly in vitro. The growth kinetics of the two types of ESCs were compared (Fig. 1C), and no difference in proliferative capacity was observed between the two $(\mathrm{P}>0.05)$. The capacity of teratoma formation of the two types of cells was then examined. Histological observation revealed that both types could form teratomas containing ganglion (ectodermal), gland (endodermal) and cartilage (mesodermal) tissue 4 weeks after subcutaneous injection in nude mice (Fig. 1D), indicating that these cells possessed pluripotent differentiation potential in vivo.

Induced mesoderm differentiation potential of J1 ESCs and pESCs in vitro. The osteogenic, chondrogenic and adipogenic differentiation potentials were tested in J1 ESCs and pESCs. At 20 days after being cultured in osteogenic induction medium, osteogenesis was indicated by the expression of osteocalcin and upregulation of alkaline phosphatase. Alizarin Red S staining further confirmed that both ESCs exhibited osteogenic potential and triggered calcium deposition (Fig. 2A). Chondrogenic differentiation was proven by gene expression of aggrecan and type II collagen. The strong staining by Safranin O could be observed in J1 ESC derivatives and pESC derivatives 20 days after induction (Fig. 2B). The adipogenic differentiation was validated by the expression of adipogenic genes, including fatty acid-binding protein 4 (fabp4) and peroxisome proliferator-activated receptor $\gamma(\operatorname{ppar} \gamma)$, and Oil red $\mathrm{O}$ staining confirmed the formation of lipid droplets in the cytoplasm (Fig. 2C). The study of gene expression and matrix production revealed that both J1 ESCs and pESCs were able to differentiate toward mesoderm lineages, including osteoblasts, chondrocytes and adipocytes.

pESCs exhibited a lower adipogenic differentiation capability compared with J1 ESCs. The adipogenic potential of J1 ESCs and pESCs was further compared systematically. The scheme of adipogenic differentiation is shown in Fig. 3A. The mRNA levels of adipogenic-specific and stemness markers during the process were first compared using qPCR. As shown in Fig. 3B, the expression of adipogenic-specific genes increased gradu- 
A
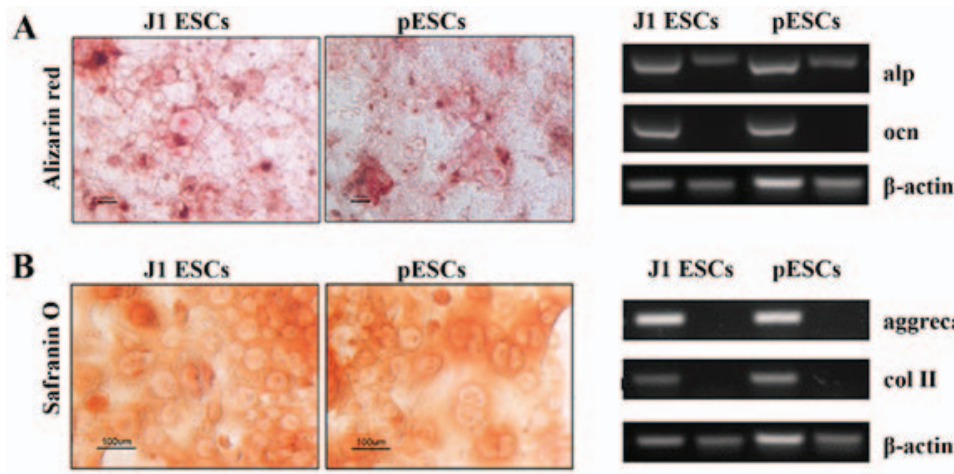

J1 ESCs pESCs

C
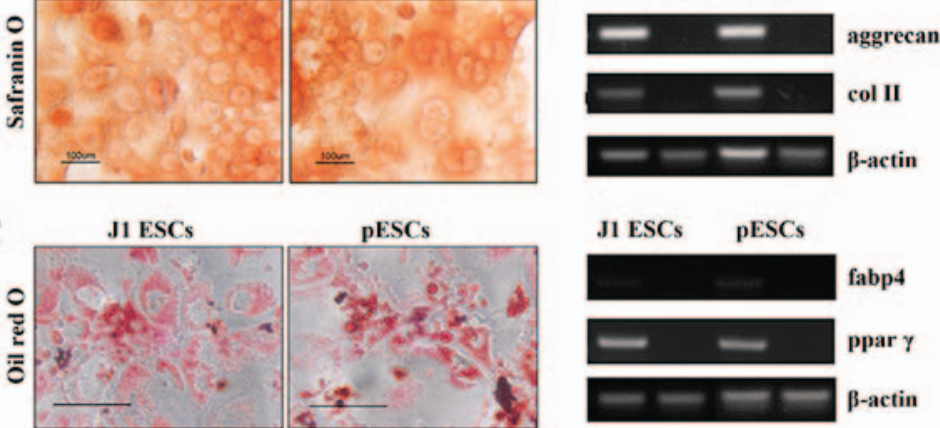

Figure 2. Multilineage potential of J1 embryonic stem cells (ESCs) and parthenogenetic ESCs (pESCs). (A) The expression of alkaline phosphatase (alp) and osteocalcin (ocn) by reverse transcription-polymerase chain reaction (RT-PCR) confirmed osteogenic differentiation 14 days after osteogenic induction. Alizarin Red staining signified mineralized deposits at day 14. (B) RT-PCR confirmed chondrogenic differentiation with expression of aggrecan and type II collagen 14 days after chondrogenic induction. Proteoglycans were stained by Safranin O on day 14. (C) RT-PCR confirmed adipogenic differentiation by the expression of fatty acid binding protein 4 (fabp4) and peroxisome proliferator-activated receptor $\gamma(\operatorname{ppar} \gamma)$ within 20 days of culture in adipogenic induction medium. Oil red O staining for lipid-containing adipocytes was detected on day 20. Scale bar, $100 \mu \mathrm{m}$.

A

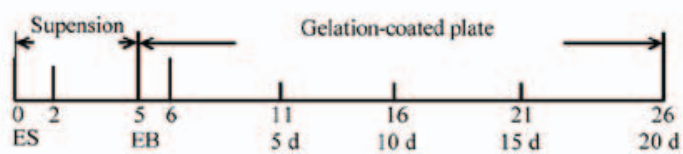

B
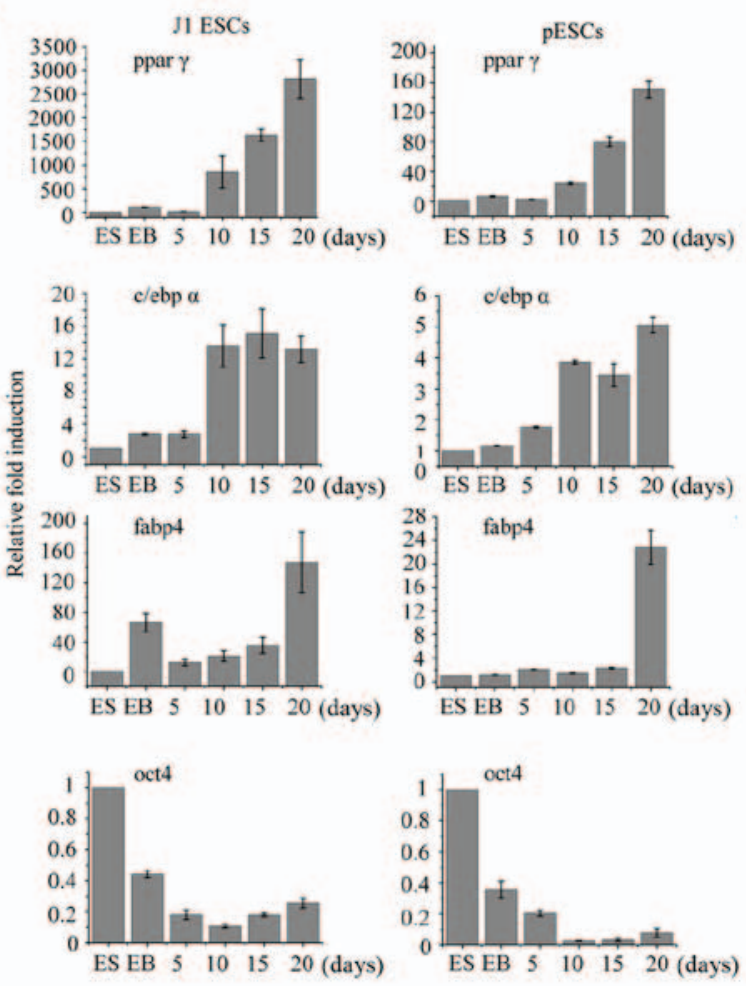

\section{C}

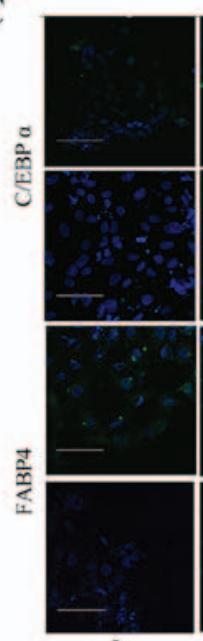

5

D

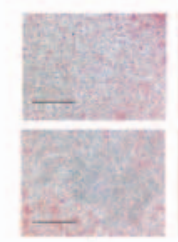

5

$\mathbf{E}$

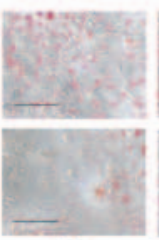

10

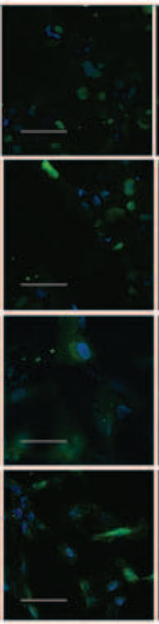

15

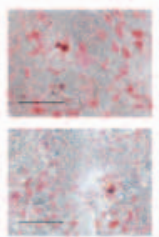

15

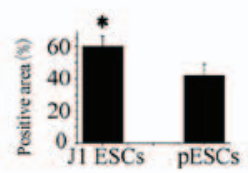

Figure 3. Adipogenesis of J1 embryonic stem cells (ESCs) and parthenogenetic ESCs (pESCs). (A) Scheme used for ESC differentiation into adipocytes (B) Adipogenic differentiation of J1 ESCs and pESCs was confirmed by upregulation of CCATT/enhancer-binding protein $\alpha$ (c/ebp $\alpha$ ), fatty acid-binding protein 4 (fabp4) and peroxisome proliferator-activated receptor $\gamma$ (ppar $\gamma$ ), and downregulation of oct 4 by quantitative polymerase chain reaction at the ES and EB stages, at 5, 10, 15 and 20 days (3 repeat/timepoint; data represent means \pm standard deviation). (C) Immunofluorescence labeling of adipogenic-specific factors c/ebp $\alpha$ and fabp4 during adipogenesis of J1 ESCs and pESCs in adipogenic induction medium at 5, 10, 15 and 20 days. (D) Image of Oil red O showing fat droplet formation at 5, 10, 15 and 20 days during adipogenesis of J1 ESCs and pESCs. (E) Statistical analysis of Oil red O-positive cells. Scale bar, $100 \mu \mathrm{m}$. ES, embryonic stem; EB, embryoid body. 

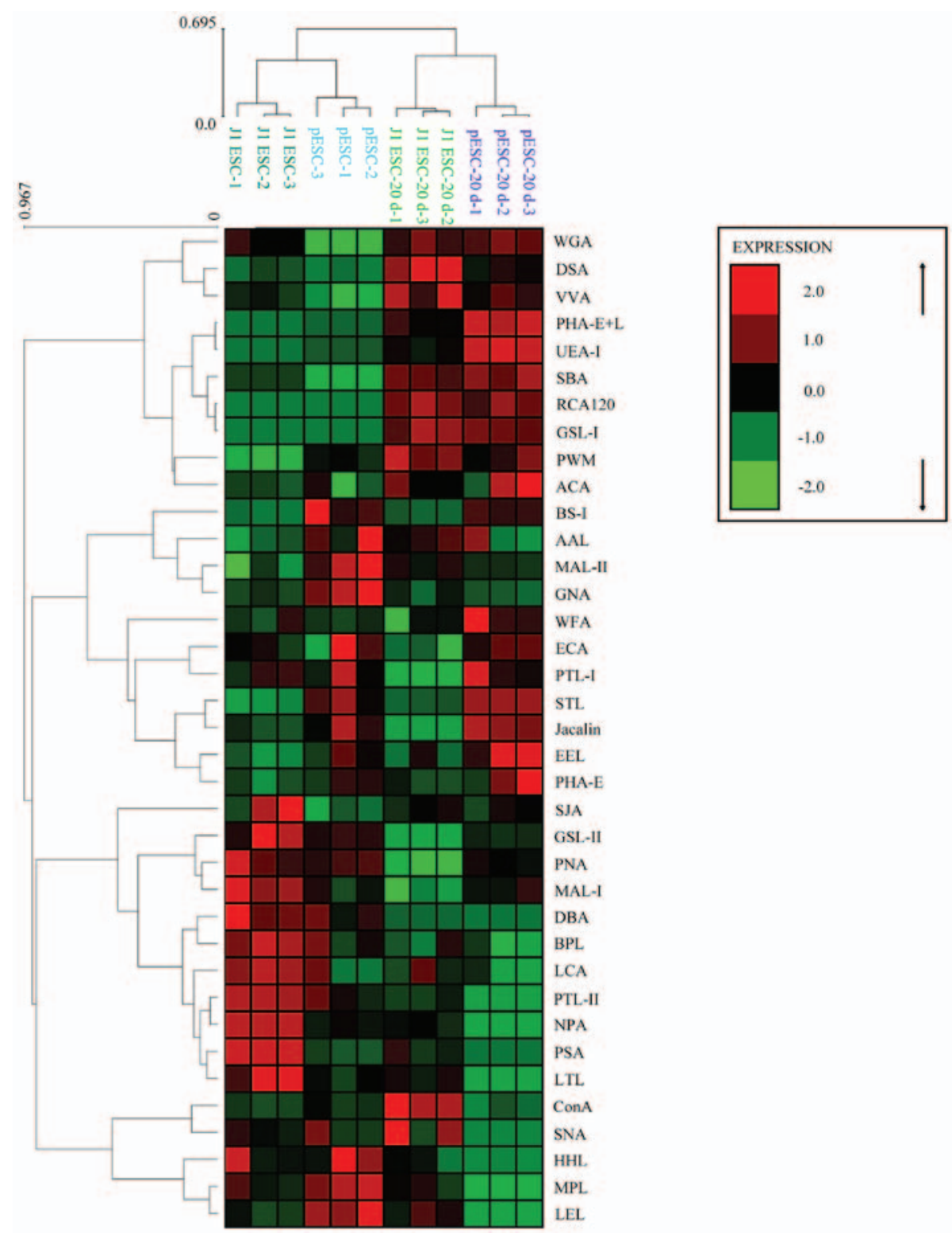

Figure 4. Heat map and hierarchical clustering. Lectin microarray data of 37 lectins of J1 embryonic stem cells (ESCs) and parthenogenetic ESCs (pESCs) during adipogenesis (ES and EB stages, 5, 10, 15 and 20 days) were mean-normalized then analyzed by Expander 6.0 software. The color indicates expression levels relative to other data in the row. Red, high; green, low; black, medium. ES, embryonic stem; EB, embryoid body.

ally, and that of stemness marker Oct4 decreased gradually during adipogenic differentiation. At 20 days after induction, the expression of ppar $\gamma$, c/ebp $\alpha$ and fabp4 was upregulated 2,757-, 13- and 147-fold, respectively, in J1 ESC derivatives, and 151-, 5- and 23-fold, respectively, in pESC derivatives. The cells were then incubated with antibodies to detect adipogenic-specific protein expression during adipogenesis. The results demonstrated that c/ebp $\alpha$ and fabp4 were both gradually upregulated during the process (Fig. 3C). However, the expression of c/ebp $\alpha$ and fabp 4 could be detected in J1 ESCs derivatives as early as 5 days after induction, while it could only be detected in pESC derivatives 10 days after induction. Both cell types could be induced to differentiate into adipocytes accompanied by increased accumulation of lipids (Fig. 3D). Cell counting revealed that, after 20 days of induction, $60.4 \pm 6.4 \%$ and $42.2 \pm 6.9 \%$ Oil red O-positive cells were observed in J1 ESC and pESC derivatives, respectively (Fig. 3E; $\mathrm{P}<0.05$ ). Collectively, these results indicate that

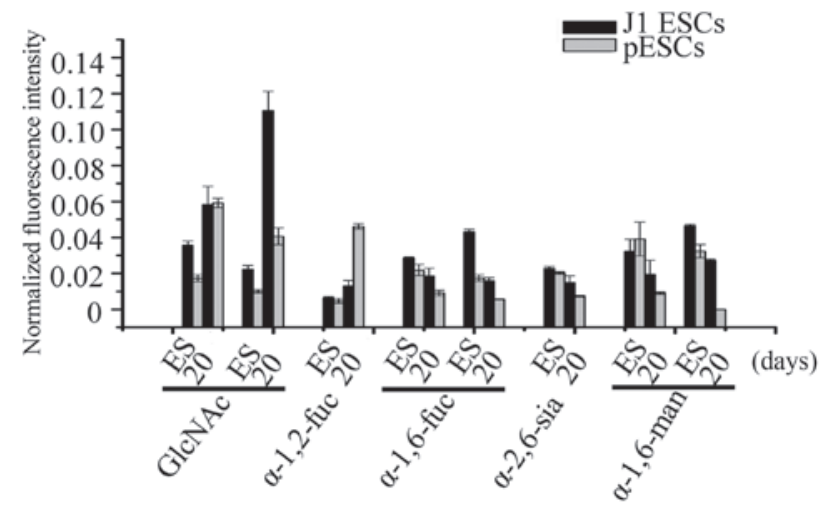

Figure 5. Similar carbohydrate chain alterations were detected by the lectin microarray. The normalized fluorescence intensity of wheat germ agglutinin, Datura stramonium agglutinin and Ulex europaeus agglutinin-1 were increased in both types of embryonic stem cells (ESCs), while the normalized fluorescence intensities of Lens culinaris, Pisum sativum and Sambucus nigra agglutinins, Hippeastrum hybrid lectin and Narcissus pseudonarcissus agglutinin were decreased. 
A a

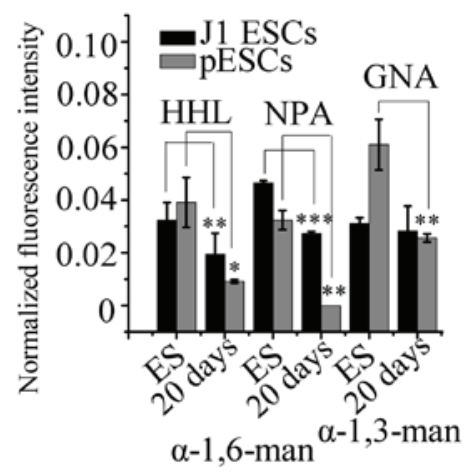

B a

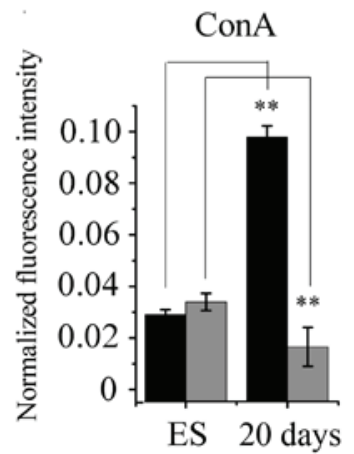

b

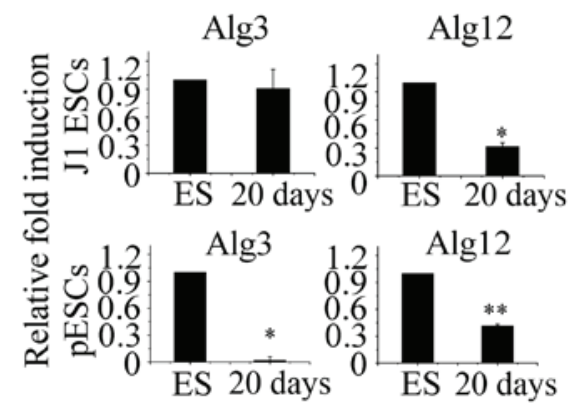

b

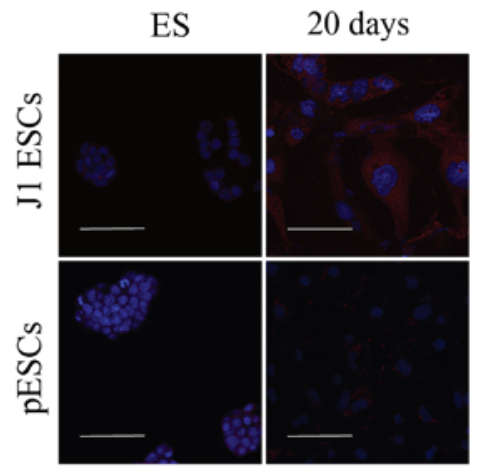

\section{C}

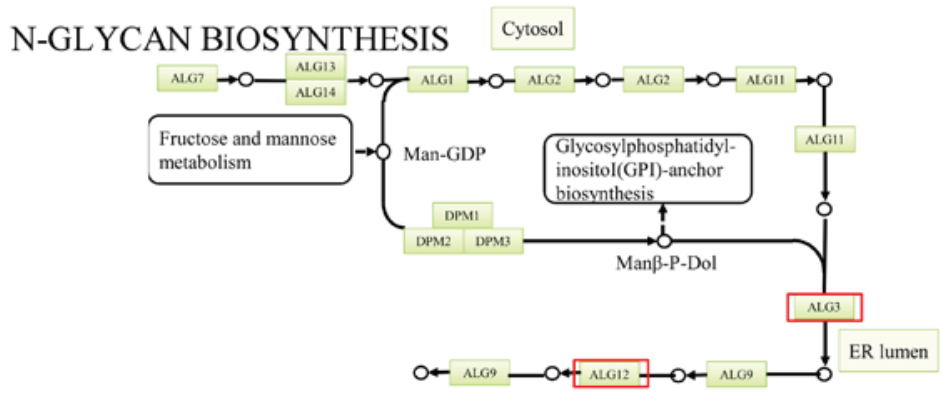

Figure 6. Protein glycosylation analysis in J1 embryonic stem cells (ESCs) and parthenogenetic ESCs (pESCs). (A) Alteration of mannosylation level of ESCs during adipogenesis. (B) Concanavalin A (ConA) was analysed by lectin microarray and lectin histochemistry. ES, undifferentiated ESCs; 20 days, differentiated ESCs. ${ }^{*} \mathrm{P}<0.05,{ }^{* *} \mathrm{P}<0.01,{ }^{* * *} \mathrm{P}<0.001$. (C) Biosynthetic pathway of N-glycan precursors. The transcription level of Alg12 was decreased in J1 ESCs and pESCs during adipogenesis, while Alg3 was decreased in pESCs (marked with red frame).

pESCs exhibited a lower adipogenic potential compared with J1 ESCs.

Glycosylation alteration of J1 ESCs and pESCs during adipogenesis. Lectin microarray chips containing 37 lectins were used to screen the alterations of protein glycosylation of J1 ESCs and pESCs during adipogenesis. The data were analyzed by hierarchical clustering after being mean-normalized. As shown in Fig. 4, J1 ESCs/pESCs and their adipogenic lineage derivatives were clearly separated into two large clusters, indicating that undifferentiated ESCs and their derivatives exhibited specified glycan profiles.

After 20 days of adipogenic differentiation, 21 of the 37 lectins used in the lectin microarray exhibited a similar trend of alteration in J1 ESCs and pESCs, among which the signal of 7 lectins increased, such as $\alpha-1-2$-fucose-binding lectin Ulex europaeus agglutinin-1 (UEA-1), GlcNAc-binding lectins wheat germ agglutinin (WGA) and Datura stramonium agglutinin (DSA). The signal of another 12 lectins decreased, including $\alpha-1-6$-fucose-binding lectins Lens culinaris agglutinin (LCA) and Pisum sativum agglutinin (PSA), a-2-6-sialic-acid-binding lectin Sambucus nigra agglutinin

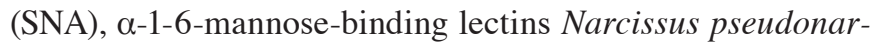
cissus (NPA) and Hippeastrum hybrid lectin (HHL) (Fig. 5). In addition, the signals of all 4 mannose-binding lectins we used [NPA, HHL, Galanthus nivalis agglutinin (GNA) and concanavalin A (ConA)] were decreased in pESCs. The signals of $\alpha-1-6-$ mannose-binding lectins (NPA and HHL) were decreased, and the signal of $\alpha-1-3$-mannose-binding lectin (GNA) exhibited no significant alteration in J1 ESCs (Fig. 6A-a). The signal of ConA was increased in J1 ESCs (Fig. 6B-a). The signals of the other GlcNAc lectins (identified with DSA) were largely increased during adipogenesis in J1 ESCs (4.95-fold) and pESCs (4.02-fold) (Fig. 5). 
The expression of glycosyltransferases was consistent with the results of the lectin microarray. The expression of $\alpha$-1-6-mannosyltransferase (Alg12) was decreased in J1 ESCs $(0.35$-fold $)$ and pESCs $(0.42$-fold $)$. The expression of $\alpha-1-3$-mannosyltransferase (Alg3) was decreased in pESCs (0.02-fold), while there was no significant alteration in J1 ESCs (Fig. 6A-b). The lectin histochemistry of ConA was consistent with the results of the lectin microarray. ConA displayed strong binding to the central and/or perinuclear cytoplasm in J1 ESCs and pESCs. After 20 days of adipogenic differentiation, the binding intensified in J1 ESC derivatives. However, ConA exhibited a similar binding intensity in pESC derivatives compared with undifferentiated pESCs (Fig. 6B-b). Alg12 was decreased during adipogenesis of ESCs, while Alg3 was decreased in pESCs. Alg3 and Alg12 are involved in the biosynthesis of the $\mathrm{N}$-glycan precursor, suggesting that the synthesis of N-glycans may decrease (Fig. 6C).

\section{Discussion}

In the present study, the alterations in the protein glycosylation of ESCs during adipogenesis were investigated. The level of GlcNAc residue and $\alpha-1-2$-fucosylation increased, $\alpha$-1-6-fucosylation, $\alpha-2-6$-sialylation and $\alpha-1-6$-mannosylation decreased in J1 ESCs and pESCs, whereas $\alpha$-1-3-mannosylation decreased in pESCs, while there was no significant alteration in J1 ESCs during adipogenesis. The protein glycosylation of ESCs was significantly altered during adipogenesis. J1 ESCs and pESCs exhibited obvious differences in protein glycosylation during adipogenesis. To the best of our knowledge, this is the first study to investigate alterations of protein glycosylation in pESCs during adipogenesis.

Protein glycosylation, a ubiquitous post-translational modification, exerts crucial biological effects on protein function (28). Protein glycosylation often modulates protein function to affect several biological processes, including development, differentiation, cell adhesion and cell-cell recognition. Indeed, heparin sulfate proteoglycans have been reported as regulatory factors of ESCs (29).

ESCs may differentiate into adipocytes using an adipogenic cocktail containing IGF-1, glucocorticoids and cAMP. Over the past few decades, adipogenic differentiation has been extensively investigated (3-5). However, the alterations in protein glycosylation during this process remain unknown. In the present study, we analysed the alterations in the protein glycosylation of J1 ESCs and pESCs during adipogenesis using lectin microarray. Among the 37 lectins, 21 exhibited the same alterations in J1 ESCs and pESCs. $\alpha-2-6-$ Sialic acid (identified by SNA) was decreased, which was in agreement with a previous study reporting that the expression of 2-6-sialylated glycan is higher in undifferentiated cells (30). It was also observed that $\alpha-1-6$-mannose (identified by HHL and NPA) was decreased during adipogenesis. All these data indicate that J1 ESCs and pESCs exxhibited similar alterations in protein glycosylation during adipogenesis. All these glycan structures may be used as promising stem cell biomarkers.

Mammalian oocytes may be parthenogenetically activated under appropriate stimuli, leading to the development of diploid non-embryonic blastocysts (31). The pluripotent stem cells from this type of blastocoel inner cell mass are defined as pESCs, which lack paternal imprints. pESCs are well histocompatible (32) and their use is not associated with ethical limitations. In the present study, J1 ESCs and pESCs exhibited similar fundamental biological properties. Both cells were able to form teratomas with three germ layers in vivo and differentiate into multiple mesenchymal tissues, including bone, cartilage and fat. These data were in agreement with the findings reported by previous studies $(26,33)$ and suggested that pESCs may be an attractive source for cell therapy. Chen et al used pESC-derived MSCs for adipogenic differentiation (26). After 3 weeks of adipogenic induction, approximately $10 \%$ and $<1 \%$ Oil red O-positive cells were observed in the ESC-derived and pESC-derived cells, respectively. In the present study, the proportion of Oil red O-positive cells was up to $60 \pm 6.4 \%$ in J1 ESC derivatives and $42 \pm 6.9 \%$ in pESC derivatives, indicating that $\mathrm{pESCs}$ have a lower adipogenic potential. In addition, 20 days after induction, the upregulated level of adipocyte genes in pESCs (ppar $\gamma, 151$-fold; c/ebp $\alpha, 5$-fold; and fabp4, 23-fold) was lower compared with that in J1 ESCs (ppar $\gamma, 2,757$-fold; c/ebp $\alpha, 13$-fold; and fabP4, 147-fold); c/ebp $\alpha$ and fabp 4 could not be detected in pESC derivatives by immunofluorescence analysis 5 days after induction.

Of the 37 lectins used in the lectin microarray, the majority exhibited the same alterations in J1 ESCs and pESCs during adipogenesis. The signal of WGA (identiying multivalent sialic acid and GlcNAc residue) was increased in J1 ESCs and pESCs during adipogenesis, whereas the signal of ConA (identiying GlcNAc residue and $\alpha$-mannose) was increased in J1 ESCs and decreased in pESCs. The level of GlcNAc residue (identified by DSA) was increased in J1 ESCs and pESCs during adipogenesis. This demonstrated that level of $\alpha$-mannose identified by ConA in pESCs was decreased. Four types of mannose-binding lectins were differentially altered in J1 ESCs and pESCs during adipogenesis. The signals of NPA and HHL (identiying $\alpha-1-6$ mannose) were decreased in J1 ESCs and pESCs. The signal of GNA (identiying $\alpha-1-3$ mannose) was only decreased in pESCs, but exhibited no significant alterations in J1 ESCs $(\mathrm{P}>0.05)$. These data suggested that the increased signal of ConA was caused by the increased level of GlcNAc residue but not $\alpha$-mannose. These findings were further confirmed by the expression profiles of glycosyltransferases. The expression of $\operatorname{Alg} 12$ ( $\alpha-1-6$ mannosyltransferase) was upregulated in J1 ESCs and pESCs. The expression of Alg3 ( $\alpha-1-3$ mannosyltransferase) was only downregulated in pESCs, but exhibited no significant alterations in J1 ESCs. The decreased expression of $\mathrm{Alg} 3$ and 12 are involved in the biosynthesis of the N-glycan precursor. This may be associated with the process of adipogenesis.

In conclusion, in the present study, it was observed that protein glycosylation in ESCs was altered similarly during adipogenesis. The similar alterations in J1 ESCs and pESCs may be used as glycobiology markers of ESCs during adipogenesis. Furthermore, J1 ESCs and pESCs exhibited obvious differences in protein glycosylation during adipogenesis. In conclusion, the precise protein glycosylation alterations associated with ESC adipogenesis was analyzed and validated, which may enable a better understanding of adipogenesis in ESCs, and may also act as markers for monitoring adipogenic development. 


\section{Acknowledgements}

This study was supported by the National Natural Science Foundation, China (grant nos. 31270126 and 31300797) and the Natural Science Basic Research Plan in Shaanxi Province of China (grant no. 2013JC2-03).

\section{References}

1. Shepherd PR, Gnudi L, Tozzo E, Yang H, Leach F and Kahn BB: Adipose cell hyperplasia and enhanced glucose disposal in transgenic mice overexpressing GLUT4 selectively in adipose tissue. J Biol Chem 268: 22243-22246, 1993.

2. Wolfgang MJ and Lane MD: Control of energy homeostasis: role of enzymes and intermediates of fatty acid metabolism in the central nervous system. Annu Rev Nutr 26: 23-44, 2006.

3. Dani C, Smith AG, Dessolin S, Leroy P, Staccini L, Villageois P, Darimont C and Ailhaud G: Differentiation of embryonic stem cells into adipocytes in vitro. J Cell Sci 110: 1279-1285, 1997.

4. Tang QQ, Otto TC and Lane MD: Commitment of C3H10T1/2 pluripotent stem cells to the adipocyte lineage. Proc Natl Acad Sci USA 101: 9607-9611, 2004.

5. Serrero G and Lepak NM: Prostaglandin F2alpha receptor (FP receptor) agonists are potent adipose differentiation inhibitors for primary culture of adipocyte precursors in defined medium. Biochem Biophys Res Commun 233: 200-202, 1997.

6. Bowers RR, Kim JW, Otto TC and Lane MD: Stable stem cell commitment to the adipocyte lineage by inhibition of DNA methylation: role of the BMP-4 gene. Proc Natl Acad Sci USA 103: 13022-13027, 2006.

7. Bowers RR and Lane MD: Wnt signaling and adipocyte lineage commitment. Cell Cycle 7: 1191-1196, 2008.

8. Spinella-Jaegle S, Rawadi G, Kawai S, Gallea S, Faucheu C, Mollat P, Courtois B, Bergaud B, Ramez V, Blanchet AM, et al: Sonic hedgehog increases the commitment of pluripotent mesenchymal cells into the osteoblastic lineage and abolishes adipocytic differentiation. J Cell Sci 114: 2085-2094, 2001.

9. van der Horst G, Farih-Sips H, Löwik CW and Karperien M: Hedgehog stimulates only osteoblastic differentiation of undifferentiated KS483 cells. Bone 33: 899-910, 2003.

10. MacDougald OA and Lane MD: Transcriptional regulation of gene expression during adipocyte differentiation. Annu Rev Biochem 64: 345-373, 1995.

11. Student AK, Hsu RY and Lane MD: Induction of fatty acid synthetase synthesis in differentiating 3T3-L1 preadipocytes. J Biol Chem 255: 4745-4750, 1980.

12. Darlington GJ, Ross SE and MacDougald OA: The role of C/EBP genes in adipocyte differentiation. J Biol Chem 273: 30057-30060, 1998.

13. Ailhaud G, Grimaldi P and Négrel R: Cellular and molecular aspects of adipose tissue development. Annu Rev Nutr 12: 207-233, 1992

14. Apweiler R, Hermjakob $\mathrm{H}$ and Sharon N: On the frequency of protein glycosylation, as deduced from analysis of the SWISS-PROT database. Biochim Biophys Acta 1473: 4-8, 1999.

15. Lowe JB and Marth JD: A genetic approach to mammalian glycan function. Annu Rev Biochem 72: 643-691, 2003.

16. Hwang HY, Olson SK, Esko JD and Horvitz HR: Caenorhabditis elegans early embryogenesis and vulval morphogenesis require chondroitin biosynthesis. Nature 423: 439-443, 2003.
17. Crocker PR: Siglecs: sialic-acid-binding immunoglobulin-like lectins in cell-cell interactions and signalling. Curr Opin Struct Biol 12: 609-615, 2002.

18. Collins BE and Paulson JC: Cell surface biology mediated by low affinity multivalent protein-glycan interactions. Curr Opin Chem Biol 8: 617-625, 2004.

19. Liu D, Shriver Z, Venkataraman G, El Shabrawi Y and Sasisekharan R: Tumor cell surface heparan sulfate as cryptic promoters or inhibitors of tumor growth and metastasis. Proc Natl Acad Sci USA 99: 568-573, 2002.

20. Sasisekharan R, ShriverZ, Venkataraman G and Narayanasami U: Roles of heparan-sulphate glycosaminoglycans in cancer. Nat Rev Cancer 2: 521-528, 2002.

21. Muramatsu $\mathrm{T}$ and Muramatsu $\mathrm{H}$ : Carbohydrate antigens expressed on stem cells and early embryonic cells. Glycoconj J 21: 41-45, 2004.

22. Qin Y, Zhong Y, Dang L, Zhu M, Yu H, Chen W, Cui J, Bian H and $\mathrm{Li} \mathrm{Z}$ : Alteration of protein glycosylation in human hepatic stellate cells activated with transforming growth factor- $\beta 1$. J Proteomics 75: 4114-4123, 2012.

23. Ito H, Kuno A, Sawaki H, Sogabe M, Ozaki H, Tanaka Y, Mizokami M, Shoda J, Angata T, Sato T, et al: Strategy for glycoproteomics: identification of glyco-alteration using multiple glycan profiling tools. J Proteome Res 8: 1358-1367, 2009.

24. Yu H, Zhu M, Qin Y, Zhong Y, Yan H, Wang Q, Bian H and Li Z: Analysis of glycan-related genes expression and glycan profiles in mice with liver fibrosis. J Proteome Res 11: 5277-5285, 2012.

25. Hwang NS, Kim MS, Sampattavanich S, Baek JH, Zhang Z and Elisseeff J: Effects of three-dimensional culture and growth factors on the chondrogenic differentiation of murine embryonic stem cells. Stem Cells 24: 284-291, 2006.

26. Chen Y, Ai A, Tang ZY, Zhou GD, Liu W, Cao Y and Zhang WJ: Mesenchymal-like stem cells derived from human parthenogenetic embryonic stem cells. Stem Cells Dev 21: 143-151, 2012.

27. Scillitani G, Zizza S, Liquori GE and Ferri D: Lectin histochemistry of gastrointestinal glycoconjugates in the greater horseshoe bat, Rhinolophus ferrumequinum (Schreber, 1774). Acta Histochem 109: 347-357, 2007.

28. Moremen KW, Tiemeyer M and Nairn AV: Vertebrate protein glycosylation: diversity, synthesis and function. Nat Rev Mol Cell Biol 13: 448-462, 2012

29. Sasaki N, Okishio K, Ui-Tei K, Saigo K, Kinoshita-Toyoda A, Toyoda H, Nishimura T, Suda Y, Hayasaka M, Hanaoka K, et al: Heparan sulfate regulates self-renewal and pluripotency of embryonic stem cells. J Biol Chem 283: 3594-3606, 2008.

30. Satomaa T, Heiskanen A, Mikkola M, Olsson C, Blomqvist M, Tiittanen M, Jaatinen T, Aitio O, Olonen A, Helin J, et al: The $\mathrm{N}$-glycome of human embryonic stem cells. BMC Cell Biol 10: 42, 2009.

31. Paffoni A, Brevini TA, Gandolfi F and Ragni G: Parthenogenetic activation: biology and applications in the ART laboratory. Placenta 29 (Suppl B): 121-125, 2008.

32. Kim K, Lerou P, Yabuuchi A, Lengerke C, Ng K, West J, Kirby A, Daly MJ and Daley GQ: Histocompatible embryonic stem cells by parthenogenesis. Science 315: 482-486, 2007.

33. Didié M, Christalla P, Rubart M, Muppala V, Döker S, Unsöld B, El-Armouche A, Rau T, Eschenhagen T, Schwoerer AP, et al: Parthenogenetic stem cells for tissue-engineered heart repair. J Clin Invest 123: 1285-1298, 2013.

This work is licensed under a Creative Commons Attribution-NonCommercial-NoDerivatives 4.0 International (CC BY-NC-ND 4.0) License. 